\section{Transnasal Aerosol Delivery to Pediatric Patients: Jet Versus Vibrating Mesh}

\section{To the Editor:}

In the recent paper by El Taoum and associates, ${ }^{1}$ an in vitro comparison of aerosol dose delivery using jet and vibrating mesh nebulizers with a variety of nasal interface devices in pediatric patient models was presented. The results of this study demonstrated that the jet nebulizer was more efficient than the vibrating mesh nebulizer across all of the nasal interface devices tested. In the conclusion, the authors stated that "Careful pairing of the aerosol generator and interface is very important during transnasal aerosol delivery." This inarguable fact, however, was not evident in the design or results of this otherwise admirable study.

The vibrating mesh nebulizer system used in the study is engineered, recommended, and licensed for use via a mouthpiece or aerosol mask patient interface, not by the nasal route, in pediatric patients. Practical use of any vibrating mesh technology in this setting would require a supplemental flow of gas to drive the aerosol particles toward the patient interface and therefore made available for inhalation. The failure to use a supplemental gas flow with the vibrating mesh nebulizer places the performance of the vibrating mesh technology at an inherent disadvantage compared with a jet nebulizer.

In my personal correspondence with one of the authors, it was implied that the intent of this study was to demonstrate which nasal interface devices might be more efficient in the home care setting for pediatric patients. In this context, it can be appreciated and understood that the study results imply that jet nebulization might be the preferred method for certain pediatric patients. However, readers of the Journal may not appreciate the unstated study intent and design limitations and may come away with the wrong message regarding the use of vibrating mesh nebulization in this patient population. Careful pairing of the aerosol generator, patient interface, and the target patient is extremely important during any evaluation of aerosol delivery, a fact that was overlooked in the design of this study and the publication of its results.

Mark S Siobal RRT-ACCS FAARC Respiratory Care Services
San Francisco General Hospital San Francisco, California

Mr Siobal discloses a relationship with Aerogen.

DOI: $10.4187 /$ respcare.04399

\section{REFERENCE}

1. El Taoum KK, Xi J, Kim J, Berlinski A. In vitro evaluation of aerosols delivered via the nasal route. Respir Care 2015;60(7):10151025 .

\section{Transnasal Aerosol Delivery to Pediatric Patients: Jet Versus Vibrating Mesh-Reply}

\section{In Reply:}

We thank Mr Siobal for his interest in our work. ${ }^{1}$ It is frequently said that children are not small adults, and aerosol drug delivery is not an exception. Infants and young children are not obligate nose breathers by choice but because of the anatomical and physiological properties of their upper airways. Even when a face mask is used as an interface for aerosol delivery, this age group will inhale the aerosol through the nose. Reports generated using oral delivery in non-anatomically correct models have led to an overestimation of the amount of drug delivered to the lung. The strength of our data is based on the fact that we showed good in vitro/in vivo correlation. ${ }^{1}$

Mr Siobal is critical of the study design and claims that the vibrating mesh technology is being unfairly evaluated. We respectfully disagree with his point of view. Our report was not intended to be a trial of the vibrating mesh technology. Our study compared lung delivery of two different types of commercially available nebulizers using different interfaces in an anatomically correct model. As stated above, members of this age group are obligate nose breathers irrespective of the interface. Mr Siobal states that "Practical use of any vibrating mesh technology in this setting would require a supplemental flow of gas to drive the aerosol particles toward the patient interface and therefore make them available for inhalation." However, Mr Siobal's opinion is not in agreement with the manufacturer's own submission for clearance to the FDA. The device (Aeroneb Go, Aerogen, Mountain
View, California) was cleared by the FDA using the $510(\mathrm{k})$ route. $^{2}$ This process compares the proposed device with other similar devices that have already been cleared. The device was compared with the Pari LC Star and Omron MicroAir, which do not use supplemental gas flow. The device received the following indications for use: "The Aeroneb Go nebulizer, for use by pediatric and adult patients, is intended to aerosolize physician-prescribed solutions for inhalation that are approved for use with a general-purpose nebulizer." There is neither mention of any specific interface that has to be used nor mention of the need to use a supplemental gas source. However, even if the interfaces were not specifically approved, the reality is that practitioners might think of using them. Our data will hopefully discourage them from doing this because of the low lung deposition.

In addition, Ari et $\mathrm{al}^{3}$ reported that the use of supplemental air flow ( $2 \mathrm{~L} / \mathrm{min})$ to a vibrating mesh nebulizer and special reservoir during mouthpiece delivery decreased lung delivery by $>50 \%$ in an adult model of a spontaneously breathing subject. Their data also suggested that mask design played a critical role in optimization of lung delivery. Moreover, there were no differences between a jet nebulizer and a vibrating mesh nebulizer used with a special reservoir and receiving $2 \mathrm{~L} / \mathrm{min}$ of external gas flow using a model of a spontaneously breathing child.

Mr Siobal underestimates the readership by stating that they could be confused about who the subject of this study is. The first sentence of the Background section of the abstract clearly defines the population of interest (infants and young children). In addition, the journal provides a section called Quick Look that provides the reader with current knowledge and the contribution of the paper. A review of that section clearly sets the framework of the study that practitioners are about to read.

Ariel Berlinski MD

Department of Pediatrics College of Medicine

University of Arkansas for Medical

Sciences

Pediatric Aerosol Research Laboratory

Arkansas Children's Hospital Research Institute

Little Rock, Arkansas 